Studies have shown that reduction to allergen exposure can reduce allergic symptoms. In the home these allergies are primarily caused by house-dust mites, pets and tobacco smoke.

\section{Case 2}

A young mother who lived with her two sons. She had always denied her asthma: she did not take her inhaled steroids, she smoked, was overweight and took no exercise. After the intervention period, her attitude towards her asthma had changed: she complied with her medication and kept a peak flow chart; she was reducing her cigarette smoking, taking exercise and had joined a diet club.

Without objective measurements i.e. PEF readings, people with asthma could over or under estimate the severity of their asthma. Breathlessness caused by being unfit or overweight could be interpreted as 'asthma' without this objective measurement.

\section{Case 3}

One lady, aged 56, who lived with her partner was on step 5 asthma treatment from her general practitioner. There had never been any PEF records to back up the need for all this medication. Since keeping a record chart this lady's treatment has been reduced from step 5 to step 4 of the asthma management in accordance with the British Guidelines on Asthma Management. ${ }^{11}$

There was a general agreement that keeping a PEF chart was helpful for both the patient and the nurse. The patients agreed that thinking about their asthma and keeping a diary of their feelings helped them in controlling their asthma symptoms. Self-management of their asthma came higher in the patients' list of priorities at the end of the intervention period.

Conclusions

Although the numbers are very small, the results indicate that the intervention programme was effective in encouraging patients to develop their own priorities for managing their asthma and reducing the risk of asthma exacerbations. Home visits provided a more relaxed atmosphere which was more appropriate for this type of psychological intervention than a busy outpatient clinic. All the patients were grateful for the one-to-one attention which ensured that some one listened to their views.

This was a small pilot study but the results support the use of this type of intervention and further studies would be justified.

\section{Acknowledgements}

We thank the Astra Foundation for their generous sponsorship which allowed us to carry out this pilot study. I thank Alison Neale, Marion Jacques, Kathy Gould and Teresa Barker for their help and skills throughout this pilot study, and Dr Brian Harrison for his continuing support.

\section{References}

1. Harrison B D W, Pearson M G. Audit in acute severe asthma - who benefits? J R Coll Physicians Lond 1993; 27: 387-90.

2. Campbell D A, Yellowlees P M, McLennan G et al. Psychiatric and medical features of near fatal asthma. Thorax 1995; 50: $254-9$.

3. Kirscht J P, Kirscht J S, Rosenstock I. A test of intervention to increase adherence to hypertensive medical regimens. Health Educ $Q$ 1981; 261-72.

4. Yoon R, McKenzie D K, Bauman A et al. Controlled trial evaluation of an asthma education programme for adults. Thorax 1993; 48: 110-16.

5. Zigmond A S, Snaith R P. The hospital anxiety and depression scale. Acta Psychiatr Scand 1983; 67: 361-70.

6. Goldberg D P, Williams P. A users guide to the general health questionnaire. Windsor, NEFR Nelson 1988.

7. Pilowsky I, Spence N. Manual for the Illness Behaviour Questionnaire. Adelaide, University of Adelaide 1994.

8. Rand: 36 item Health Survey. 1.0 Rand Health Sciences Programme. Santa Monica, C A Rand Corporation 1992.

9. Wallston B S, Wallston K A, Kaplan G D et al. Development and validation of the Health Locus of Control (HLC) Scale. J Consult Clin Psychol 1976; 44: 580-5.

10. Hyland M M. The living with asthma questionnaire. Resp Med 1991; 85: 13-6 (Suppl B).

11. The British Thoracic Society, The National Asthma Campaign, The Royal College of Physicians of London et al. British guidelines on asthma management. Thorax 1997; 52: S1-21.

\title{
Evaluating Asthma Audit - experiences from practice
}

\author{
G Hoskins, R G Neville and B Smith
}

\begin{abstract}
Objectives: To elicit how a clinical audit package is used within general practice and explore perceptions of audit as an educational tool.

Design and subjects: Semi-structured interview of health professionals within eight selected general practices who participated in a National Audit of Asthma Management.

Results: The audit process was found to be valuable in highlighting the strengths and weaknesses in asthma management. In seven out of the eight practices the lead person conducting the audit was a nurse. The most common problems encountered
\end{abstract}

were time constraints, incomplete information in the notes and lack of a common working practice. Three practices complained of patients who did not attend for assessment, one complained that the audit had been imposed on her and two found the randomisation procedure difficult to follow. The feedback was found to be fair, relevant and thought provoking. Proposed changes were identified including updating of asthma lists and of recall systems, improved information recording, and an increased role and more training for nurses. The importance of repeating the audit was acknowledged. 
Conclusion: Clinical care of patients with asthma is enhanced by a multi-disciplinary approach. Audit of asthma care should likewise involve shared responsibilities and decision making by practice nurses and GPs.

\section{INTRODUCTION}

The 1990 NHS reforms stressed the importance of audit as a 'routine' procedure to achieve and maintain a high standard of health care provision.

The prime reason for performing audit is to improve patient care while maximising the use of resources. ${ }^{2}$ The advantages are well documented ${ }^{3-6}$ with Health Authorities actively encouraging health professionals to undertake audit by providing audit facilitators backed by financial assistance. The publication of distance learning material such as the 'Moving to Audit' programme, is aimed at introducing audit into the workplace.?

To be successful audit must be relevant to clinical practice, linking educational activity to everyday situations of direct relevance to patient management. Asthma is a 'marker condition' of practice clinical care in which there is a key role for the practice nurse. An increasing number of practice nurses have specialist asthma training ${ }^{8}$ allowing them a major role in asthma care. However, the growth in practice nursing has not been supported by a professional nursing infrastructure and they continue to have a minor role in policy decision making. There is a need to audit the provision of asthma care in general practice and the influence of a trained asthma nurse.

Informed decision making leads to better quality care but successful audit demands time and, if it is to be associated with good practice, participants have to be convinced of the real benefits it can bring. To encourage practices to conduct regular audit procedural problems must be overcome. Therefore, there is a need to explore whether the success of practice nurse and GP collaboration on clinical care of asthma extends to success with implementing audit of asthma care. This paper reports on how practices utilised an 'off the peg' clinical audit package and explores doctors' and nurses' perceptions of working on audit within their practice.

\section{Method}

The National Asthma Management Study ${ }^{9}$ was an audit-based distance learning package designed to encourage audit of asthma management in general practice and to examine the structures available for the provision of asthma care. Two hundred and twenty five practices from throughout the United Kingdom completed a questionnaire on a pre-determined sample of 30 patients from their asthma register. The patients were then invited to attend the surgery for assessment of their current symptoms. On return of the recording booklet to the research unit a personalised critique of asthma management was prepared based on the British Thoracic Society (BTS) guidelines for the management of asthma and aimed at both the nurse and the doctor. The comments were non-judgemental. Typical comments included:

'Have you considered issuing a self-management plan?' 'Guidelines suggest that patients taking excessive amounts of $\beta_{2}$-agonists should have a step up in medication.'
'Well controlled patients can have their treatment stepped down.'

Detailed instruction on how to proceed with the audit and enrol patients was provided and throughout the project a telephone helpline was available to answer queries from the practices. The study (reported elsewhere) demonstrated that measures of outcomes can be attained by using a more streamlined system of practice based audit. ${ }^{9}$

Participants were asked to fill in an evaluation form on completion of the audit. This indicated a high satisfaction rating for the package. To gauge the efficacy of this approach to audit and the feedback given, a small number of participants were interviewed personally. Information on the way they carried out the audit and the problems which arose during the process was obtained. Participant practices were grouped into urban or rural, large (greater than three partners) or small and a representative mix of eight within one day's travel of the investigator $(\mathrm{GH})$ were selected for an indepth visit.

This large study required data quality assurance checks and visits to ensure participating professionals were adhering to the study protocol. These visits also presented an opportunity to explore qualitative aspects of how practices cope with the problems and challenges of audit. A semi-structured interview schedule was constructed to give participants a chance to voice their views and perceptions of how they and their practices felt about involvement in an audit of their asthma care. A series of open-ended questions were used as a basis for interview (Table 1). Transcripts and taping were omitted and reporting was done immediately after interview by the interviewer $(\mathrm{GH})$.

The interviews took place after the practices had completed the data collection phase of the study on asthma management and received patient specific feedback; but before completion of a complete audit cycle.

\section{RESULTS}

Participating health professionals in the eight practices selected were all receptive to an interview. In two of the practices the participating doctor was interviewed alone, one having completed the audit by himself, the other having 'supported' the practice nurse who was unavailable at the time of the interview. In three practices the nurse was interviewed alone and in three both the doctor and the practice nurse were present at the interview.

Participants from all eight practices reported that they found the practical aspects of the study straightforward but two of the nurses reported difficulty in understanding the instructions for the patient selection procedure, which used a random number sequence. In seven practices the lead person conducting the study was the practice nurse.

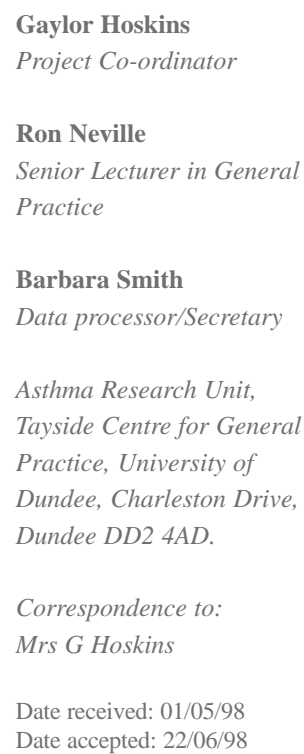


Two had made the decision themselves to participate, the other five having been asked by their GP. The eighth was carried out by a general practitioner who did not employ a practice nurse.

Two practices reported that they had no difficulty completing the study and audit, one also reported having encountered no problems during the process. The most common difficulty reported was the length of time it took to complete although this was, in most cases, less time than specified in the briefing documents. Participants also reported that there was a problem in persuading other members of the primary care team to participate. This became an important issue when implementing changes brought about by the audit findings.

Practical problems encountered included incomplete information from notes, whether written or computerised, in five practices. A typical comment was,

'Finding out the number of prescriptions each patient had in the last 12 months was difficult and time consuming. The practice is not yet computerised for this function and the written notes were not always up-to-date.'

Three interviewees expressed problems with patients not attending for clinical assessment, 'Getting people to attend for clinical assessment is hard, asthmatics who think they are well will not come in for a review.'

Participants from five out of the eight practices represented reported lack of a common working practice with follow-up not always being arranged and incomplete recording:

'Although we are computerised there are gaps in the information with the computer not always updated if a prescription is given manually.'

'After initial attack treatment not all the patients are asked to come back to the surgery and not all patients are being referred to the nurse for follow-up.'

Administration problems were experienced by four practices,

'As yet I do not have a computer console for my own use although this is to change.'

'Help in collecting the data and calling the patients in for assessment was not available.' and in one practice the nurse felt she was given little choice but to participate in the study,

'I had no choice in doing this audit, the doctor who thought it would be a good idea lost interest and it landed on my desk.'

Participants were asked to give both their initial assessment of the feedback provided and their opinion after having had time to study and reflect.

Initial reactions were mixed admitting that their first impression of the audit critique was that it was 'generalised', 'over critical', 'condescending', 'inaccurate' and 'made the practice look inefficient'. In three practices both the doctor and the practice nurse were present during the interview and had initially reacted differently to the feedback.

\section{Practice One}

Nurse - 'When I first read the feedback my initial reaction was that it was over-critical but on balance I realise why.'

Doctor - 'The feedback was very generalised but the very nature of the process means it can't be anything else.'

\section{Practice Two}

Nurse - 'I thought the feedback was very detailed and fair.'

Doctor - 'Without looking at the recording booklet and the reasons why some of the comments were generated I thought some were inaccurate but I have knowledge of patients' treatment obviously not in the notes.'

\section{Practice Three}

Nurse - 'My first impression was that some of the comments were awful, I thought they made me look incompetent.'

Doctor - 'I actually thought it made the practice look good and I found the feedback very useful.'

The practice nurse who felt 'pressurised' into carrying out the audit said;

'At first I have to admit to being angry at the comments but they were certainly thought provoking and definitely food for thought.'

An experienced asthma nurse who carried out the study and audit independently stated:

'My first impression was that they were condescending e.g. "Symptoms stable, can a step down in medication be considered?" I thought "how stupid" if we can do that we would. However, on reflection I realise that not everyone is as experienced.'

After study of the feedback, recommendations from the British Thoracic Society Asthma Guidelines, and the patients notes, all the interviewees were ultimately satisfied with the audit package. Presentation was deemed 'detailed and fair'. An 'identifiable theme' was evident throughout which proved 'thought provoking and relevant'.

The interviewees all reported having learned some lessons from the process not least the 'value of audit'. They acknowledged that simple things were not being done and administrative procedures needed tightening up with prescription recording and recall procedures needing improvement. Clinic times needed to be reviewed and medication reduction should be considered more often with more patients receiving written self-management plans. Some comments were as follows.

'Counting the prescriptions was a good exercise and has led to a tightening up in the recording procedure.' 'More self-management plans need to be given.' 'The practice has had fewer emergency nebulisations/hospital contacts/home visits in the last few years, the audit highlighted this trend.' 'Simple things are not being done e.g. peak expiratory flow, inhaler technique, regular review.' 'Partnership with other health professionals and administration/clerical staff needs to be improved if future audits are to be more easily carried out.' 
All interviewees reported proposed changes in the practice management of asthma for the near future which included updating of asthma lists and recall systems, computerisation of prescription procedures, regular use of a recording stamps, introduction of open clinic by appointment and an increased role for the practice nurse with more nurses being offered training.

The participants, including the nurse who had not independently chosen to undertake the audit, appeared keen to close the 'audit loop' by repeating the process in the following year.

\section{DisCUSSION}

The process of audit is time consuming and audit data currently collected by Health Authorities is often only for administrative purposes. Practices, therefore, have a right to object to procedures which require time but do not necessarily have a direct impact on the improvement of patient care. The National Asthma Management Study audit ${ }^{9}$ was designed to allow practices to examine their management of asthma and, with the aid of feedback, effect change in their clinical practice and improve patient care. The booklet served as a simple clinical audit and its strength was that it represented what was actually being done rather than what is said to be done.

For pragmatic reasons the interview was conducted after the 'study' phase of the project but before completion of an 'audit loop'. In retrospect, an interview after the completion of an audit loop may have yielded better insights into health professionals views. A further criticism is that the methodology used to illicit views was added on to a practice visit to monitor study protocol adherence. A stand alone qualitative project might have elicited more insights into nurses and doctors opinions and attitudes to audit.

Some of the problems associated with audit within general practice were highlighted during the interviews. In only three practices the audit was completed by the person who made the decision to undertake it. In seven practices the entire workload was carried out by one person, the practice nurse. In some cases administrative back up was unavailable. Difficulty in retrieving information from patients notes was also experienced. 'Lack of a common working practice' highlighted by some of the interviewees indicates the need for clinical audit which focuses the minds of all members of the health care team.

Agreement on the advantage of the procedure is essential if dedicated time is to be set aside for carrying it out. Without this, acceptance of the results may prove difficult and a barrier to the creation of change and participation in future clinical audit. To encourage participation audit must be seen as a tool for improving clinical practice and in turn patient care and, as such, should be a team effort and not the responsibility of a single individual. Ownership of the audit by the participants is important, ${ }^{7}$ sharing the workload increases this and may lead to better communication between not only members of the health care team but all employees in the practice. This joint participation may, in turn, increase the likelihood that any changes highlighted by the feedback are more likely to be implemented and that audit becomes an integral part of general practice.

The necessity of selecting a representative sample of patients using a random numbers sequence although understood, was an area of contention. Audit must be designed to look at the complete picture, the good as well as the bad, only then can changes which will be effective be made. Hence the importance of using a randomised procedure to select the patients. The difficulty found by some in understanding this simple statistical methodology highlighted the importance of clear 'easy to follow' instructions, supported by a 'helpline'.

A measure of the success of the audit may be that the practices were not always comfortable with the feedback, but all accepted it and expressed a wish to move forward and implement changes in their management procedures. It had been a valuable exercise which highlighted simple things which needed to be done. Five of the practices interviewed completed the 'audit cycle' the following year. The three who did not, cited lack of dedicated time, rather than lack of enthusiasm. The experiences gained from this 'off the peg' audit may encourage others in preparing similar packages for use in general practice.

\section{Conclusion}

Interviews with participants from an audit project showed that clinical audit is considered a valuable exercise. Nurses are prepared to perform clinical audit but need support from all members of the primary health care team and protected time to complete the task.

\section{RECOMMENDATIONS}

1. Practices who enrol in audit need to negotiate this with all members of the primary care team.

2. If practice nurses are to be encouraged to audit asthma care they need support and protected time.

\section{References}

1. Sec of State for Health. Medical Audit Working Paper 6. HMSO 1989; ISBN 01/321216X: 6.

2. Defining essential data for audit in general practice. $B M J$ 1990; 300: 92-4.

3. Chidley K E, Wood-Baker R, Town G I et al. Reassessment of asthma management in an accident and emergency department. Resp Med 1991; 85: 373-7.

4. Lipworth B J, Jackson C M, Ziyaie D et al. An audit of acute asthma admissions to a respiratory unit. Health Bulletin 1992; 50: 389-98

5. Barritt P W, Staples E B. Measuring success in asthma care: a repeat audit. Br J Gen Pract 1991; 41: 232-6.

6. Neville R G, Clark R C, Hoskins G, et al. National Asthma Attack Audit 1991-92. BMJ 1993; 306: 559-62.

7. Centre for Medical Education, Dundee. Moving to Audit 1992. University of Dundee.

8. National Asthma and Respiratory Training Centre. Diploma in Asthma Care, Warwick.

9. Neville R G, Hoskins G, Smith B, et al. Observations on the structure, process and clinical outcomes of asthma care in general practice. Br J Gen Pract 1996; 46: 583-87. 